\title{
Recent Evidence on Worldwide Trends on Sleep Duration
}

\author{
Camilla Hoyos $^{1}$ • Nick Glozier ${ }^{1,3}$ - Nathaniel S. Marshall ${ }^{1,2}$
}

Published online: 8 October 2015

(C) Springer International Publishing AG 2015

\begin{abstract}
It is often claimed that we are living through a global sleep loss epidemic where, as a society, we increasingly get less and less sleep. However, our previously published systematic review of all relevant studies until May 2011 failed to find strong evidence that this had happened worldwide. In this current review, we updated that search and found 5 new articles with data from 12 countries starting in the 1960s1980s and culminating in 2001-2012. We still find little evidence for the claimed epidemic as different countries have increasing, decreasing or stable sleep. There remain strong concerns about methodological quality in many of the studies and the effect of the rise of smart phones and tablets cannot yet be assessed. We also found data in 5 reports about 4 countries where markers of sleep quality do seem to have declined.
\end{abstract}

Keywords Sleep $\cdot$ Insomnia $\cdot$ Secular trends $\cdot$ Prevalence

\section{Introduction and the 2012 Review}

Insufficient sleep is a clear public health threat through its effects on occupational or traffic-related injuries, lost productivity, impaired mood and possibly important chronic diseases

This article is part of the Topical Collection on Sleep Epidemiology

Nathaniel S. Marshall

nathaniel.marshall@sydney.edu.au

1 NHMRC Centre for Integrated Research and Understanding of Sleep (CIRUS), Woolcock Institute of Medical Research, University of Sydney, Sydney, Australia

2 Sydney Nursing School the University of Sydney, Sydney, NSW 2006, Australia

3 Brain and Mind Centre, University of Sydney, Sydney, Australia such as diabetes, cancer and obesity [1]. When describing this problem in the media or even in peer-reviewed articles, the sleep loss problem is referred to as an epidemic (reviewed in $[2,3])$. However, the term 'epidemic' is used in infectious disease epidemiology to indicate a sudden sharp rise in the number of cases of a disease. Applying this to sleep behaviour implies therefore that society as a whole is getting less sleep than it used to. Intuitively for some reason this feels 'right' somehow. But where is the evidence for this having happened? Who did it happen to? For instance, are there vulnerable sub-populations?

In attempting to find the evidence for this occurring worldwide in the adult population, our previously published review on this topic sought to find all suitable articles published up until May 2011 [2]. Suitable studies were those which had a clear population-based sampling strategy so that the data could be reasonably assumed to be representative of community-dwelling adults. These mostly turned out to be nationally representative sampling frames but we included smaller regional or occupational studies as long as they met the study design requirements. Sleep duration could be assessed by any one of a number of methods (questionnaire, time-use survey, etc.), but the same technique had to be repeated in cross-sectional surveys with the same sampling frame. This was so that we could reasonably be sure that any changes were due to a real change in prevalence rather than some effect of a change in the measurement of sleep or in the types of people being asked. Cohort studies (where groups of people are followed up longitudinally) are not suitable for this question because any population changes in sleep behaviour would be inseparable from the ageing process because the same people would be older at each follow-up. Survivor bias in any follow-up of a cohort is also going to lose all the people who have died or been lost to follow-up because they are too unwell to participate. 
Our review [2] found 12 suitable study reports with data from 15 countries (including 4 studies from the United States). Interestingly, the majority of the studies used time-use survey data, conceptually and pragmatically similar to sleep diary data. Time-use data are generally collected by government departments for the purposes of understanding changes in important social forces such as commuting time and paid and unpaid working hours. Some studies reported proportions of people sleeping below 5 or $6 \mathrm{~h}$ but most reported the mean value change (and many helpfully reported both). The results were notably heterogeneous. In 6 countries sleep duration had declined (Austria, Belgium, Finland, Germany, Japan and Siberia in Russia). But the rates of change were very small with the declines reported being $\leq 0.6 \mathrm{~min}$ per year. Different data sources from the United States indicated both increases and decreases in sleep duration and the data from Goteborg in Sweden was similarly ambiguous. Conversely Britain, Bulgaria, Canada, France, Korea, the Netherlands and Poland all reported increased average sleep duration over the past decades. But again these gains were extremely modest with most countries gaining less than a minute per year except for Korea (1.5 min per year) and Bulgaria (1.7 min per year). We could not tell why this heterogeneity exists between countries as the methodological rigour, the general culture of the countries, the timeframe of the reports (over decades from the 1960s to the 2000s) or the number of follow-ups seemed similar between countries with increasing and decreasing sleep duration. We concluded that there was no consistent evidence to support the claim of a worldwide sleep loss epidemic. Even in the United States, we could find no compelling evidence of a societal increase in sleep loss.

But what about the adolescent or young adult population? We know that cross- sectional studies have demonstrated associations between the amount of technology intrusion and shorter sleep durations [4-6]. This could represent an actual effect, or may represent activity displacement (e.g. shorter or later sleepers who used to read under the covers or listen to the radio now use mobile devices at night). Mattriciani and colleagues review paper looked at trends in sleep duration over the last 100 years in children and adolescents, that was co-published with ours [2, 3]. Although reported as showing a small decrease in sleep, overall this result was driven by a statistically significant decrease in a couple of large Southeast Asian studies, whilst Australia, United States and Northern Europe showed no decrease and possibly even a small increase in sleep duration. As such, the field needs to be careful when the data from large studies in one cultural setting mask differential trends in other settings. Quantitative combinations of heterogeneous data from multiple countries into a single estimate may in fact be misleading when trying to describe global trends.

\section{New Data Published since the Previous Review}

Since the May 2011 review census date, we have published additional time-use survey data from 10 countries [7]. We were also aware of additional repeated cross-sectional sleep data from British and Finnish sources and so it seems timely to update our review $[8,9]$. We searched all citations to our 2012 [2] and 2013 [7] articles and some other key articles in the field [10]. We also conducted a non-systematic search of PubMed and Scopus databases for papers describing trends in sleep duration at a population level published after May 2011. Table 1 lists the studies located regarding sleep duration changes. Sleep duration was defined as having changed when mean sleep duration was reported to have significantly changed and/or the proportions of either short or long sleepers had changed in a direction that is consilient with a populationlevel shift towards more or less sleep. We found 3 countries where sleep duration had decreased (Norway, Italy, Canada), 4 populations that stayed the same (Netherlands, Germany, Denmark and Geneva in Switzerland) and 4 countries where sleep seemed to have increased (United Kingdom, Australia, Sweden, Finland). Again in the United States, there were conflicting data where sleep duration over the same period of the mid-1980s to the late 2000s was reported to both decrease (both papers report data from the same study, the US National Health Interview Survey [11, 12]) and increase [7]. Of note, all studies showing an increase in sleep duration were ones where there was little chance of response bias as the focus was not on sleep duration per se but on time-use in general.

\section{So Why the Mixed Results?}

\section{Simple Questions About Sleep Duration Remain Unvalidated}

The United States provides an example of how the ascertainment of sleep duration can fundamentally reverse the result. The two papers that reported a decrease in sleep duration both reported data from an overlapping period of time (1977-2012) from the same data source, the National Health Interview Survey. Sleep duration in this study was ascertained by a single question ('On average, how many hours of sleep do you get in a 24-h period?'). Conversely, the study reporting an increase in sleep duration in the United States which used data from the same period of time (1985-2007) collected its data using the national time-use surveys. Simple questions about sleep duration have extremely poor agreement (within only about $\pm 2.5 \mathrm{~h}$ ) with diary-based methods of sleep duration ascertainment [16]. The difference between objective measures and the responses to these simple questions is also biased - the further the respondent's objective sleep duration is 


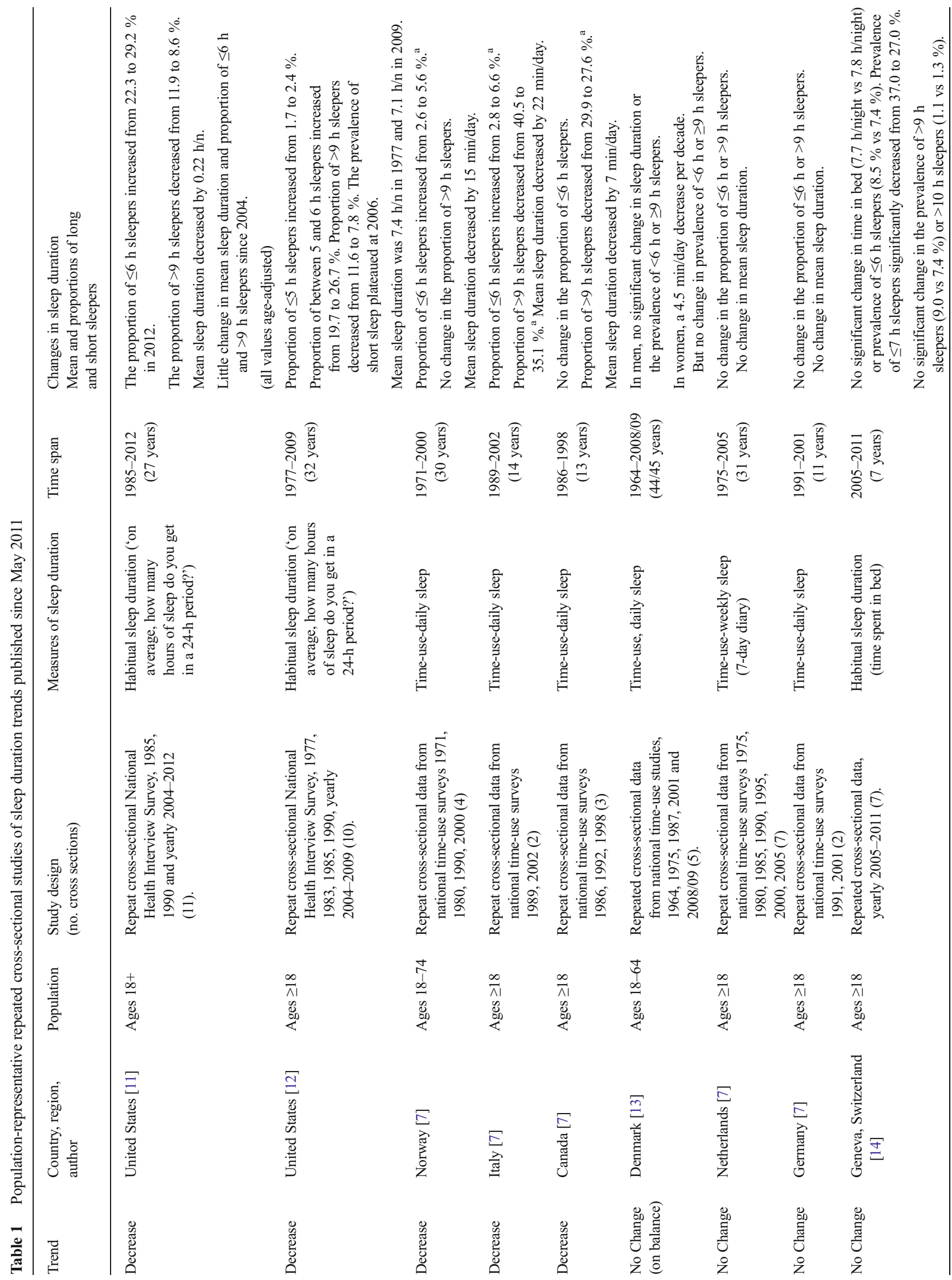




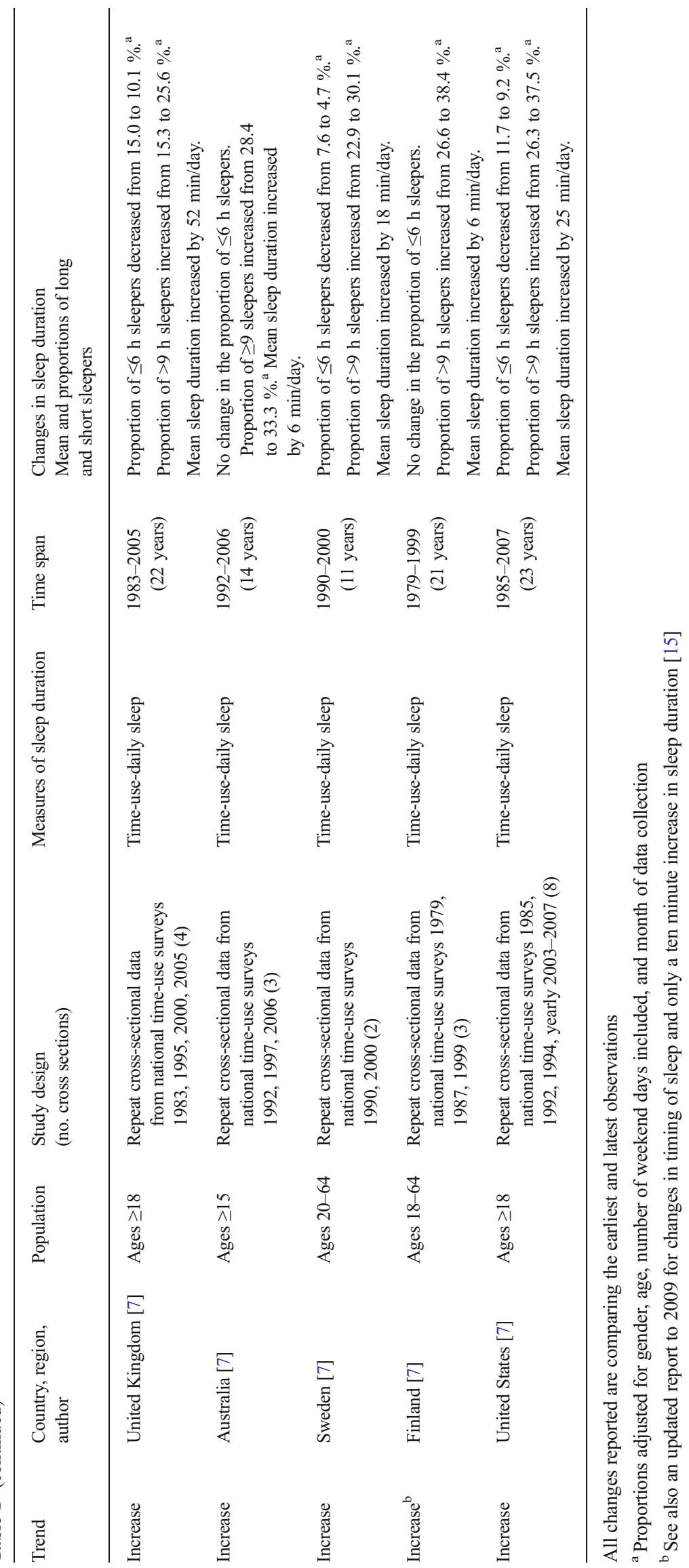


from $7 \mathrm{~h}$ the greater the discrepancy, at least in younger people with mental health issues [17]. Other studies comparing simple self-report to actigraphic measures of sleep have found only very modest correlations [18-20] but unfortunately have not reported the agreement plots. Despite their presumed 'face validity' (they appear like they should measure sleep duration), simple questions about sleep duration remain an unvalidated instrument and it is not entirely clear what exactly is being measured by them [21].

\section{The Limits of Accuracy — the Dangers of False Precision}

The Canadian time-use surveys [7] provide another useful lesson in the limits of accuracy using these techniques. The Canadian data indicated an average decrease of 7 min of sleep duration per day over a 13-year period from 1986 to 1998 . However, the data were collected using a time-use survey that partitioned the day into 10-min parcels, which means the 13year change in sleep duration is smaller than the smallest time quotient that can be measured in any human day. Similar issues exist in the National Health Interview Survey data in the United States and potentially all other studies where sleep duration has been directly queried. Even though people in these studies can sometimes theoretically estimate their sleep duration to the closest minute, most people will generally round to the nearest hour or half an hour, and much more rarely the nearest quarter of an hour [22-24]. This natural or imposed granularity in the data should be taken into account when interpreting small changes. These may be below the ability of our tools to detect. It is important to remember that our statistical analysis packages may assure us that these changes are 'statistically significant' but it is only because we have lied to them about the data being continuous. Selfreported sleep duration is really ordinal data. Another major difficulty in interpretation is that the changes may be accounted for by measurement bias trends (e.g. rounding up vs. rounding down over time). If people are increasingly told that sleep is reducing, they may preferentially round down over time and so the observed effect actually be a result of the epidemic claims.

\section{Secular Social Forces That May Drive Sleep Duration}

Why is it that some countries are going up and some countries are going down? The answer could be that sleep duration reflects socio-economic factors that are not moving in one trend with time $[25,26]$. One of the biggest predictors of sleep duration of individuals within countries is the number of hours they work (e.g. [27]). Once an individual is working above $50 \mathrm{~h}$ per week, sleep seems to suffer. Some people may choose to work these hours for reward but others may be forced to do so in low wage economies simply to survive [28, 29]. It has also been noted at a national level that sleep duration rises and falls over time, particularly economic downturn being associated with increased sleep due to increased rates of under or unemployment. Thus the decision on the start and end year of these analyses might be crucial to the interpretation-you can see something that might be a cycle in the Danish data [13]. Starting in an economic recession and finishing in an economic boom period may give a false impression of a short sleep duration epidemic and the converse starting in a boom period and finishing in a recession might falsely give the impression that sleep duration is increasing. A similar pattern has been observed consistently in cardiovascular disease (CVD) whereby, contrary to what most would expect, CVD death rates fall during recessions and rise in boom times in developed countries, purportedly due to less work and unhealthier eating [30].

Another key driver through this mechanism will be female participation in paid employment having risen dramatically since the 1960s. If increases in paid employment are not offset by decreases in unpaid work, then we should be seeing increased sleep deprivation particularly in women. Despite this seemingly plausible effect, this does not seem to have occurred across a broad cross-section of the countries when gender-specific trends were examined [13].

Secular changes in technology use are regularly cited by sleep researchers (including ourselves) as drivers of poor sleep or poor sleep behaviour, although most studies are cross sectional [4-6]. Cohort studies are less common [31]. There is also some evidence for reverse causation where the awake time suffering from problems with getting to sleep might be filled with technology use [32]. Traditionally, it was thought that access to electronic light in general may drive sleep restriction or later bedtimes and there has been some empirical evidence for this recently. Two towns in Argentina with similar cultural backgrounds but with and without access to electric power for lighting seem to have differing sleep behaviours in the expected direction [33]. However, despite the warning surrounding the effects of increasing use of mobile technology, there does not seem to have been a massive decline in sleep across the western world though the introduction of televisions in the 1960 s and 1970 s, personal computing in the 1980s, nor the rise of the internet in the 1990s and 2000s. Many of the time-use surveys reviewed here seem to finish in the 2000s. So we are as yet unable to assess in this review whether the rise of smart phones and tablets may have fundamentally altered sleep behaviour as much as is feared because the data are all more than 5 years old at this point. National time-use surveys are often repeated on 5-year cycles so we may soon have new data.

The effects of blue light-emitting technology on sleep onset or sleep timing are yet to be studied at a population level (iPhone was released in the USA in mid-2007 and the iPad in 2010). One of the most comprehensive and up-to-date reports we found one report from Finland examining time-use survey data where they had broken down sleep onset and 
offset time between 1979 and 2009 and found a progressive shift towards later bed and rise times in the working age population whilst overall sleep time has not changed (i.e. a 10-min increase) [15]. But sleep onset timing seems to be exactly the same between 1999 and 2009 indicating that the rise of the internet had no effect. Norwegian data with cross sections at 1980, 1990 and 2000 found sleep duration to be unchanged but did find that sleep had become significantly delayed [34]. Conversely, Canadian time-use data do not find any change in sleep onset or offset between 1998 and 2005 [35]. Market saturation of these devices probably occurred after 2009, and it remains to be seen what effect these devices, if any, have had on population sleep. A major consideration in this seemingly obvious route is that the amount of light experienced during the day modulates any effect of this technology use nocturnally and so we need to keep an ecological view in interpreting laboratory studies conducted in very low light $[36,37]$.

\section{Changes in Population Subgroups}

A lack of overall population change could mask subgroup trends for example in young people who seem more susceptible to the melatonin suppression effects of light-emitting devices [38]. The dangers of investigating this could be to inflate type 1 error and accidentally find an important subgroup where sleep has declined in a particular country. So we would urge that these subgroup analyses be replicated across countries to be sure that they are real effects and that researchers think about whether changes they observe in subgroups are real given the quality of the data actually collected (see "New Data Published since the Previous Review").

\section{Safety Considerations in Advising the Public to Sleep More}

The side-effect profile of the sustained message to the public that they are not getting enough sleep and that this problem is getting worse is unknown. Telling people to sleep more might not be an effective intervention any more than telling people to lose weight has been. Sleep might not be under their control anyway [29]. We should also be careful what we wish for. Pushing the bell curve of human sleep duration to the right will inevitably increase the proportion of long-sleepers. It has been noted for some time that overly long sleep may have detrimental health effects [21]. Very few of these long sleep duration studies have employed validated measures [39], the vast majority are based on simple self-report. But those studies using actigraphy to measure sleep duration have also reported that long sleep durations are associated with poorer outcomes, e.g. worse lipid profiles [40] or even death [39]. If long sleep is a true cause of these effects (rather than an epiphenomenon of an underlying disease process), then might harm public health by untargeted advice to increase sleep.

One further side-effect of the constant message to the public that they get insufficient sleep might be an increased anxiety around sleep and function amongst vulnerable sub-populations. We might in fact be causing the observed worsening of insomnia/sleep quality. One indicator of this could be an increase in insomnia prevalence and/or increased insomnia presentations to healthcare professionals.

\section{Is Sleep Quality Declining?}

One possible explanation for the widespread feeling that we are undergoing a sleep duration epidemic could be a decline in sleep quality [8]. In an attempt to supplement our updated review of sleep duration trends, we thought readers might also appreciate a review of repeat cross-sectional studies of sleep quality that we are aware of (see Table 2). Readers should be aware that our search for these was not systematic and probably omits some important studies-Table 2 is not meant to include all studies published on this topic and is shown for illustrative purposes only. Sleep quality as a concept is harder to define than sleep duration. Declines in sleep quality might be quantified as increases in complaints, presentations and diagnoses of insomnia. They could also be found by an increase in complaints about the quality, restfulness of sleep and sleep disturbance. Sleep quality has also probably declined on the back of the obesity epidemic increasing the prevalence of sleep apnea [45]. In 4 studies that we found, from England, United States, Norway and Finland, there seemed to have been an increase in the prevalence of insomnia, insomnialike symptoms or presentation for insomnia across a range of ages (see Table 2). This could explain the widespread 'feeling' that sleep duration has declined. In the final study from the United States, it was less clear to us whether insomnia had increased or whether general population growth and increased societal interaction with the health care system could have explained the increase in the absolute number of insomnia presentations [44].

\section{What Next-Future Studies and Unanswered Questions}

Italy was one country where there does seem to have been a noticeable increase in short sleep and decrease in long sleep [7]. One of the potential interpretations of this is that the traditional siesta has been lost but that nocturnal sleep has not increased to cover the difference. However, we cannot be sure this is what has happened and we are unsure whether such an effect may have occurred in other traditional siesta cultures such as Greece or Spain. We have no data on developing 


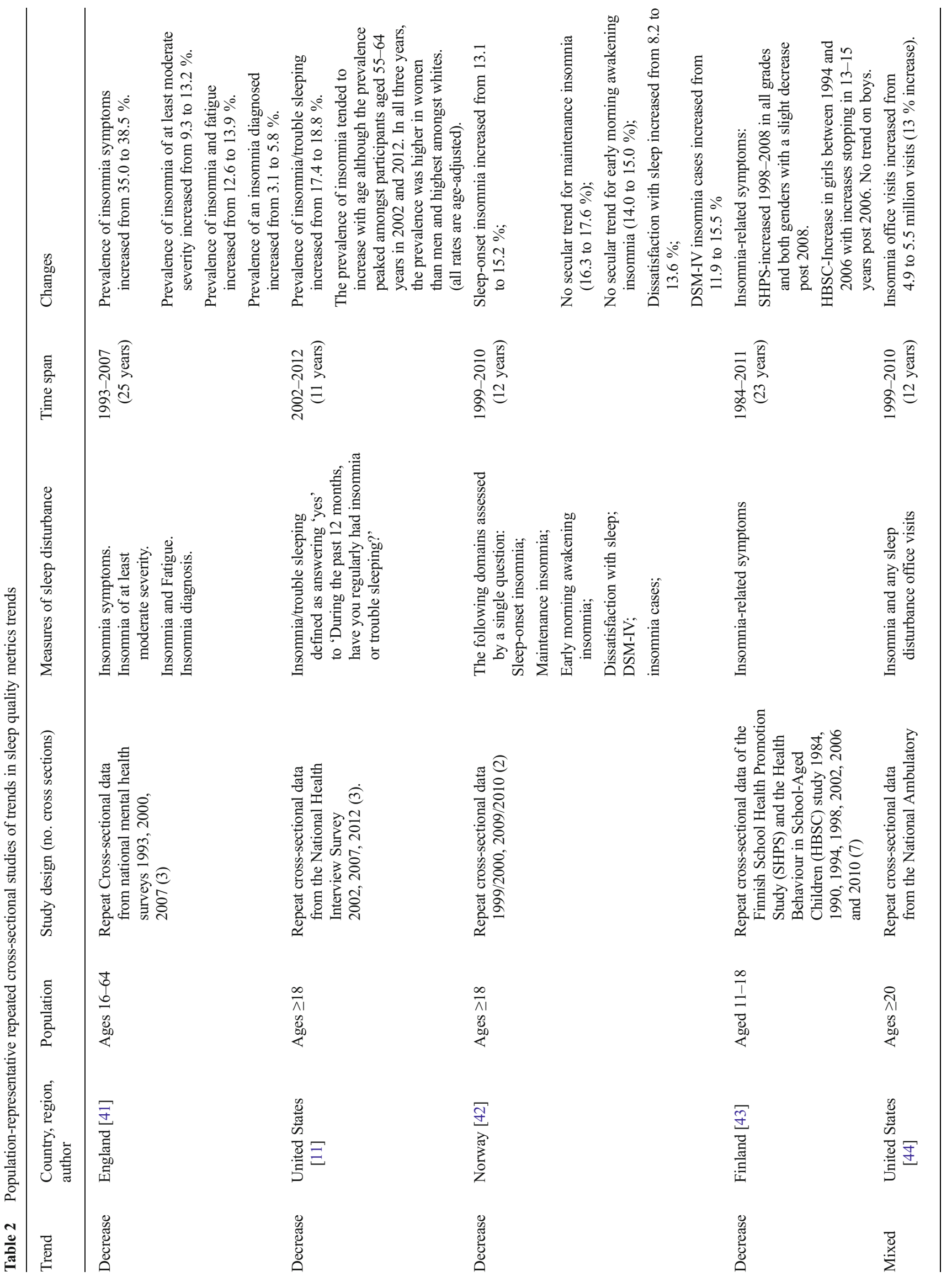


countries nor on the BRIC countries (Brazil, Russia, India and China; except for the Siberian study [46]).

We do not know whether particular sub-populations might have had a sleep duration epidemic. Vulnerable sub-populations could include 15-25-year-olds or younger (not much data). These age groups appear particularly vulnerable to technology intrusion affecting their sleep [38]. Other secular effects (work, study, extra-curricular activities both organised and un-organised) might also have imposed a tighter window for sleep than had been available in earlier decades. Bluelight-emitting devices for instance might have pushed sleep later than is traditional, but younger people cannot sleep late to make up for it because of set start times and we know that younger people with delayed sleep onset are exposed to shorter sleep durations due to these demands [47]. We have yet to systematically establish whether sleep timing has changed at a population level. Data from less established economies and countries going through economic transition are also far too sparse [48].

Because we rely upon self-report (sometimes of time-inbed), we cannot really be sure of the fidelity of the estimates of sleep duration. Objective measurements are needed - or at least some population-level validation of the time-use survey data to be sure that these are providing unbiased estimates of sleep. The ability to use technology through the enormous penetration of phone-based or wrist band accelerometers (e.g. jawbone.com/blog/2014-year-review/) may aid the field but all of these require validation. One concern is that the technology does not last long enough to be used in repeated surveys, with apps or devices being deliberately rendered obsolete through planned obsolescence after only a couple of years, undermining the basic requirement of repeated identical measures.

Declines in sleep quality should be investigated using a systematic review and any forthcoming analysis of sleep quality in objective measures should also report any effects on sleep quality/fragmentation.

\section{Conclusions}

There does not seem to have been the worldwide decline in population sleep duration (a sleep duration epidemic) that is so often claimed. There are some countries in which there is evidence of shorter sleep durations than in the past. However, there are just as many countries with apparently increasing sleep durations. Even in the United States where the idea of the sleep duration epidemic seems to be particularly fashionable, there is evidence from different sources of both a decrease and an increase in sleep duration. However, there is some evidence that there has been a decline in sleep qualitybut this needs more rigourous investigation. The field would benefit from standardised, validated and reliable approaches 
and methodology as these undermine our ability to provide good public health data and leadership

Acknowledgments Secular trends in sleep duration and health research at the University of Sydney has been supported by (NHMRC grants \#571421 \#1004528 \& \#1060992).

\section{Compliance with Ethical Standards}

Conflict of Interest Camilla Hoyos, Nick Glozier and Nathaniel Marshall have no conflict of interest or relevant financial relationships to disclose.

Human and Animal Rights and Informed Consent This article does not contain any studies with human or animal subjects performed by any of the authors.

\section{References}

1. Institite of Medicine (USA). Sleep disorders and sleep deprivation: An unmet public health problem. Washington DC: The Institute of Medicine; 2006.

2. Bin Y, Marshall N, Glozier N. Secular trends in adult sleep duration: a systematic review. Sleep Med Revs. 2012;16:223-30.

3. Matricciani L, Olds T, Petkov J. In search of lost sleep: secular trends in the sleep time of school-aged children and adolescents. Sleep Med Revs. 2012;16:203-11.

4. Chang A-M, Aeschbach D, Duffy JF, Czeisler CA. Evening use of light-emitting ereaders negatively affects sleep, circadian timing, and next-morning alertness. PNAS. 2015;112:1232-7.

5. Gamble AL, D'Rozario AL, Bartlett DJ, et al. Adolescent sleep patterns and night-time technology use: results of the Australian Broadcasting Corporation's big sleep survey. PLoS ONE. 2014;9, e111700.

6. Cain N, Gradisar M. Electronic media use and sleep in school-aged children and adolescents: a review. Sleep Med. 2010;11:735-42.

7. Bin YS, Marshall NS, Glozier N. Sleeping at the limits: the changing prevalence of short and long sleep durations in 10 countries. Am J Epi. 2013;177:826-33.

8. Glozier N. Maybe it's quality not length that matters (editorial). Sleep. 2012;35:313-4.

9. Marshall N. The sleep loss epidemic: hunting ninjas in the dark. J Sleep Res. 2015;24:1-2.

10. Knutson K, Van Cauter E, Rathouz P, et al. Trends in the prevalence of short sleepers in the USA: 1975-2006. Sleep. 2009;33:37-45.

11. Ford E, Cunningham T, Croft J. Trends in self-reported sleep duration among us adults from 1985 to 2012. Sleep. 2015;38:829-32.

12. Jean-Louis G, Williams NJ, Sarpong D, et al. Associations between inadequate sleep and obesity in the US adult population: analysis of the national health interview survey (1977-2009). BMC Public Health. 2014;14:290.

13. Bonke J. Trends in short and long sleep in denmark from 1964 to 2009 , and the associations with employment, SES (socioeconomic status) and BMI. Sleep Med. 2015;16:385-90.

14. Gubelmann C, Guessous I, Theler J-M, et al. Trends and determinants of time in bed in Geneva, Switzerland. J Clin Sleep Med. 2014;10:1129-35.

15. Pääkkönen H, Hanif R. Time use changes in Finland through the 2000s. Statistics Finland: Helsinki; 2012.

16. Miller CB, Gordon CJ, Toubia L, et al. Agreement between simple questions about sleep duration and sleep diaries in a large online survey. Sleep Health. 2015;1:133-7
17. Biddle D, Robillard R, Herms D, et al. Accuracy of self-reported sleep compared to actigraphy in young people with mental illhealth. Sleep Health. 2015.

18. Lauderdale DS, Knutson KL, Yan L, et al. Self-reported and measured sleep duration: how similar are they? Epidemiol. 2008;19: 838-45.

19. Girschik J, Fritschi L, Heyworth J, Waters F. Validation of selfreported sleep against actigraphy. J Epidemiol. 2012;22:462-8.

20. Arora T, Broglia E, Pushpakumar D, et al. An investigation into the strength of the association and agreement levels between subjective and objective sleep duration in adolescents. PLoS ONE. 2013;8, e72406.

21. Bliwise D, Young T. The parable of parabola: what the u-shaped curve can and cannot tell us about sleep. Sleep. 2007;30:1614-5.

22. Bartlett DJ, Marshall NS, Williams A, Grunstein RR. Sleep health New South Wales: chronic sleep restriction and daytime sleepiness. Intern Med J. 2008;38:24-31.

23. Gander P, Marshall N, Harris R, Reid P. Sleep, sleepiness and motor vehicle accidents: a national survey. Aust NZ J Pub Heal. 2005;29: 16-21.

24. Marshall N, Bolger W, Gander P. Abnormal sleep duration and motor vehicle crash risk (research letter). J Sleep Res. 2004;13: 177-8.

25. Brochu P, Armstrong C, Morin L-P. The 'trendiness' of sleep: an empirical investigation into the cyclical nature of sleep time. Empir Econ. 2012;43:891-913.

26. Hyyppä MT, Kronholm E, Alanen E. Quality of sleep during economic recession in Finland: a longitudinal cohort study. Soc Sci Med. 1997;45:731-8.

27. Basner M, Fomberstein KM, Razavi FM, et al. American time use survey: sleep time and its relationship to waking activities. Sleep. 2007;30:1085-95.

28. Hale L. Who has time to sleep? J Public Health. 2005;27:205-11.

29. Hale L. Inadequate sleep duration as a public health and social justice problem: can we truly trade off our daily activities for more sleep? Sleep. 2014;37:1879-80.

30. Glozier N, Tofler G, Colquhoun D, et al. The national heart foundation of Australia consensus statement on psychosocial risk factors for coronary heart disease. Med J Aust. 2013;199:179-80.

31. Thomée S, Härenstam A, Hagberg M. Computer use and stress, sleep disturbances, and symptoms of depression among young adults - a prospective cohort study. BMC Psych. 2012;12:176.

32. Tavernier R, Willoughby T. Sleep problems: predictor or outcome of media use among emerging adults at university? J Sleep Res. 2014;23:389-96.

33. de la Iglesia HO, Fernández-Duque E, Golombek DA, et al. Access to electric light is associated with shorter sleep duration in a traditionally hunter-gatherer community. J Biol Rhythms. 2015;30:34250 .

34. Sivertsen B, Øverland S, Pallesen S. Trends in timing of sleep in the general population of Norway: 1980 to 2000. Percept Motor Skills. 2011;113:509-18.

35. Michelson W. Sleep time - media hype vs Diary data. Social Indicators Research. 2011;101:275-80.

36. Chang A-M, Scheer FAJL, Czeisler CA. The human circadian system adapts to prior photic history. J Physiol. 2011;589:1095-102.

37. Zeitzer JM, Friedman L, Yesavage JA. Effectiveness of evening phototherapy for insomnia is reduced by bright daytime light exposure. Sleep Med. 2011;12:805-7.

38. Figueiro M, Overington D. Self-luminous devices and melatonin suppression in adolescents. Lighting Res Tech. 2015. doi:10.1177/ 1477153515584979

39. Kripke DF, Langer RD, Elliott JA, et al. Mortality related to actigraphic long and short sleep. Sleep Med. 2010;12:28-33. 
40. Petrov MER, Kim Y, Lauderdale D, et al. Longitudinal associations between objective sleep and lipids: the CARDIA study. Sleep. 2013;36:1587-95.

41. Calem M, Bisla J, Begum A, et al. Increased prevalence of insomnia and changes in hypnotics use in England over 15 years: analysis of the 1993, 2000, and 2007 national psychiatric morbidity surveys. Sleep. 2012;35:377-84.

42. Pallesen S, Sivertsen B, Nordhus IH, Bjorvatn B. A 10-year trend of insomnia prevalence in the adult Norwegian population. Sleep Med. 2014;15:173-9.

43. Kronholm E, Puusniekka R, Jokela J, et al. Trends in self-reported sleep problems, tiredness and related school performance among Finnish adolescents from 1984 to 2011. J Sleep Res. 2015;24:3-10.

44. Ford ES, Wheaton AG, Cunningham TJ, et al. Trends in outpatient visits for insomnia, sleep apnea, and prescriptions for sleep medications among us adults: findings from the national ambulatory medical care survey 1999-2010. Sleep. 2014;37:1283-93.

45. Peppard PE, Young T, Barnet JH, et al. Increased prevalence of sleep-disordered breathing in adults. Am J Epi. 2013;177(9): 1006-14. doi:10.1093/aje/kws342

46. Artemov V, Novokhatskaya O. Changes of time budget of the rural working population of Siberia in the 1990s. Oslo, Norway: International Association for Time Use Research; 2001.

47. Glozier N, O'Dea B, McGorry PD, et al. Delayed sleep onset in depressed young people. BMC Psych. 2014;14:33.

48. Zhang J, Marshall N, Miller C, et al. Sleep health epidemiology in low and middle income countries: A systematic review, and metaanalysis of the prevalence of poor sleep quality and sleep duration. Submitted. 2015; Under Review. 